\title{
Front Matter: Volume 8772
}

, "Front Matter: Volume 8772," Proc. SPIE 8772, Nonlinear Optics and Applications VII, 877201 (17 May 2013); doi: 10.1117/12.2030985

SPIE. Event: SPIE Optics + Optoelectronics, 2013, Prague, Czech Republic 


\title{
PROCEEDINGS OF SPIE
}

\section{Nonlinear Optics and Applications VII}

\author{
Mario Bertolotti \\ Joseph Haus \\ Alexei M. Zheltikov \\ Editors
}

\section{5-17 April 2013 \\ Prague, Czech Republic}

Sponsored by

SPIE

Cooperating Organisations

HiPER Project (United Kingdom)

ELI Beamlines (Czech Republic)

International Commission For Optics

Published by

SPIE 
The papers included in this volume were part of the technical conference cited on the cover and title page. Papers were selected and subject to review by the editors and conference program committee. Some conference presentations may not be available for publication. The papers published in these proceedings reflect the work and thoughts of the authors and are published herein as submitted. The publisher is not responsible for the validity of the information or for any outcomes resulting from reliance thereon.

Please use the following format to cite material from this book:

Author(s), "Title of Paper," in Nonlinear Optics and Applications VII, edited by Mario Bertolotti, Joseph Haus, Alexei M. Zheltikov, Proceedings of SPIE Vol. 8772 (SPIE, Bellingham, WA, 2013) Article CID Number.

ISSN: 0277-786X

ISBN: 9780819495747

Published by

SPIE

P.O. Box 10, Bellingham, Washington 98227-0010 USA

Telephone +1 3606763290 (Pacific Time) · Fax +1 3606471445

SPIE.org

Copyright @ 2013, Society of Photo-Optical Instrumentation Engineers.

Copying of material in this book for internal or personal use, or for the internal or personal use of specific clients, beyond the fair use provisions granted by the U.S. Copyright Law is authorized by SPIE subject to payment of copying fees. The Transactional Reporting Service base fee for this volume is $\$ 18.00$ per article (or portion thereof), which should be paid directly to the Copyright Clearance Center (CCC), 222 Rosewood Drive, Danvers, MA 01923. Payment may also be made electronically through CCC Online at copyright.com. Other copying for republication, resale, advertising or promotion, or any form of systematic or multiple reproduction of any material in this book is prohibited except with permission in writing from the publisher. The CCC fee code is 0277-786X/13/\$18.00.

Printed in the United States of America.

Publication of record for individual papers is online in the SPIE Digital Library.

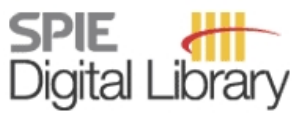

SPIEDigitalLibrary.org

Paper Numbering: Proceedings of SPIE follow an e-First publication model, with papers published first online and then in print and on CD-ROM. Papers are published as they are submitted and meet publication criteria. A unique, consistent, permanent citation identifier (CID) number is assigned to each article at the time of the first publication. Utilization of CIDs allows articles to be fully citable as soon as they are published online, and connects the same identifier to all online, print, and electronic versions of the publication. SPIE uses a six-digit CID article numbering system in which:

- The first four digits correspond to the SPIE volume number.

- The last two digits indicate publication order within the volume using a Base 36 numbering

system employing both numerals and letters. These two-number sets start with 00, 01, 02, 03, 04, $05,06,07,08,09,0 A, 0 B \ldots$. 0Z, followed by 10-1Z, 20-2Z, etc.

The CID Number appears on each page of the manuscript. The complete citation is used on the first page, and an abbreviated version on subsequent pages. Numbers in the index correspond to the last two digits of the six-digit CID Number. 


\section{Contents}

ix Conference Committee

\section{ANISOTROPIC MATERIALS}

877203 Measurement of the circular dichroism in the second harmonic optical signal produced by Au covered self ordered dielectric nanospheres [8772-2]

A. Belardini, A. Benedetti, M. Centini, G. Leahu, F. Mura, S. Sennato, E. Fazio, C. Sibilia, Univ. degli Studi di Roma La Sapienza (Italy); V. Robbiano, Univ. degli Studi di Genova and CNISM (Italy); M. C. Giordano, C. Martella, D. Comoretto, F. Buatier de Mongeot, Univ. degli Studi di Genova (Italy)

\section{NONLINEAR MATERIALS}

877208 Angular-tuning of optical parametric generation efficiency in 2D periodically poled lithium tantalate [8772-8]

M. Lazoul, Lab. des Systèmes Électroniques et Optroniques, Ecole Militaire Polytechnique (Algeria), and Lab. de Physique des Lasers, CNRS, Univ. Paris 13 (France); L. M. Simohamed, Lab. des Systèmes Électroniques et Optroniques, Ecole Militaire Polyłechnique (Algeria); L.-H. Peng, National Taiwan Univ. (Taiwan); A. Fischer, A. Boudrioua, Lab. de Physique des Laser, CNRS, Univ. Paris 13 (France)

877209 Self-diffraction of laser beams in the case of resonant excitation of excitons in colloidal CdSe/ZnS quantum dots [8772-9]

V. Dneprovskii, A. Smirnov, M. Kozlova, M.V. Lomonosov Moscow State Univ. (Russian Federation)

$8772 \mathrm{OB}$ Nonlinearly chirped quasi-phase-matching for production of ultra-broadband twin photons [8772-11]

A. R. Tamazyan, G. Y. Kryuchkyan, Yerevan State Univ. (Armenia) and Institute for Physical Researches (Armenia)

8772 OC Ultra-broadband, mid IR and coherent supercontinuum generated in aperiodic chalcogenide photonic crystal fibers [8772-12]

R. Cherif, A. Baili, M. Zghal, Univ. of Carthage (Tunisia)

\section{PLASMONICS}

8772 OG Nonlinear optical properties of Au nanoparticles in solution [8772-16]

M. Trejo-Durán, Univ. de Guanajuato (Mexico); D. Cornejo-Monroy, Univ. Autonoma de Queretaro (Mexico); E. Alvarado-Méndez, A. Olivares-Vargas, J. M. Estudillo-Ayala, Univ. de Guanajuato (Mexico); V. Castaño-Meneses, Univ. Nacional Autónoma de Mexico (Mexico) 
$8772 \mathrm{OH} \quad$ Generation of entangled polaritons in doped media [8772-17]

I. O. Barinov, A. V. Prokhorov, S. M. Arakelian, Vladimir State Univ. (Russian Federation)

SOLITONS

8772 OK Introduction of optical Newton cradle model for understanding the $\mathbf{N}$-solitons fission process under the action of higher order dispersion [8772-20]

R. Driben, Tel-Aviv Univ. (Israel) and Univ. Paderborn (Germany); B. A. Malomed, Tel-Aviv Univ. (Israel); D. V. Skryabin, Univ. of Bath (United Kingdom); A. V. Yulin, Univ. de Lisboa (Portugal)

$8772 \mathrm{OL}$ The optical control of spatial dissipative solitons in optical fibers filled with a cold atomic gas [8772-21]

M. Yu. Gubin, A. V. Prokhorov, Stoletov's Vladimir State Univ. (Russian Federation);

M. G. Gladush, Institute of Spectroscopy (Russian Federation); A. Leksin, S. M. Arakelian, Stoletov's Vladimir State Univ. (Russian Federation)

\section{APPLICATIONS/DEVICES I}

$87720 \mathrm{~N}$ Influence of non-maxwellian electron distribution in low-Z elements iluminated by a high intensity x-ray lasers [8772-23]

A. G. de la Varga, Univ. Politécnica de Madrid (Spain); F. de Gaufridy, Institute of Physics (Czech Republic) and Instituto de Fusión Nuclear, Univ. Politécnica de Madrid (Spain); P. Velarde, M. Cotelo, D. Portillo, A. Barbas, A. González, Univ. Politécnica de Madrid (Spain); P. Zeitoun, Lab. d'Optique Appliquée, CNRS, Ecole Polytechnique ParisTech (France)

877200 Experimental study of a crystalline-resonator based optoelectronic oscillator [8772-24] P. Salzenstein, A. Coillet, R. Henriet, L. Larger, Y. K. Chembo, CNRS, Institut Franche Comté Electronique Thermique Optique Sciences et Technologie (France)

8772 OP Nonlinear self-reflection of intense ultra-wideband femtosecond pulses in optical fiber [8772-25]

L. S. Konev, Y. A. Shpolyanskiy, SPbNRU ITMO (Russian Federation)

\section{APPLICATIONS/DEVICES II}

8772 OR Silicon nitride waveguide with flattened chromatic dispersion [8772-27]

J. M. Chavez Boggio, D. Bodenmueller, T. Fremberg, R. Haynes, M. M. Roth, Leibniz-Institut für Astrophysik Potsdam (Germany); R. Eisermann, L. Zimmermann, Leibniz-Institut für innovative Mikroelektronik (Germany); M. Bohm, Univ. of Potsdam (Germany)

8772 OT Numerical model for DGD estimation in optical transmission system [8772-28] J. Litvik, D. Benedikovic, Univ. of Zilina (Slovakia); M. Wuilpart, Univ. of Mons (Belgium); M. Dado, M. Kuba, Univ. of Zilina (Slovakia) 
8772 OU Double-frequency Brillouin fiber lasers [8772-29]

V. V. Spirin, Univ. of Mons (Belgium), and Scientific Research and Advanced Studies Ctr. of Ensenada (Russian Federation); C. A. López-Mercado, Scientific Research and Advanced Studies Ctr. of Ensenada (Mexico); D. Kinet, Univ. of Mons (Belgium); E. A. Zlobina, S. I. Kablukov, Institute of Automation and Electrometry, Siberian Branch (Russian Federation); P. Mégret, Univ. de Mons (Belgium); I. O. Zolotovskiy, Ulyanovsk State Univ. (Russian Federation); A. A. Fotiadi, Univ. of Mons (Belgium), loffe Physical-Technical Institute (Russian Federation), and Ulyanovsk State Univ. (Russian Federation)

8772 OV Tunable multiwavelength quantum dot external-cavity lasers [8772-30] C.-H. Pai, G. Lin, National Chiao Tung Univ. (Taiwan)

$87720 \mathrm{~W}$ Analytical model of ground-state lasing phenomenon in broadband semiconductor quantum dot lasers [8772-31]

V. V. Korenev, A. V. Savelyev, St. Petersburg Academic Univ. (Russian Federation), and St.Petersburg State Polytechnical Univ. (Russian Federation); A. E. Zhukov, St. Petersburg Academic Univ. (Russian Federation), St.-Petersburg State Polytechnical Univ. (Russian Federation), and loffe Physico-Technical Institute (Russian Federation); A. V. Omelchenko, St. Petersburg Academic Univ. (Russian Federation), and St.-Petersburg State Polytechnical Univ. (Russian Federation); M. V. Maximov, St. Petersburg Academic Univ. (Russian Federation), St.-Petersburg State Polytechnical Univ. (Russian Federation), and loffe Physico-Technical Institute (Russian Federation)

MATERIALS I

$87720 Z$ DNA translocation through a periodically patterned nanoprobe [8772-34]

S. S. Choi, M. J. Park, Sun Moon Univ. (Korea, Republic of); N. K. Park, Seoul National Univ. (Korea, Republic of); S.-M. Park, L. Lee, Univ. of California at Berkeley (United States)

\section{MATERIALS II}

877211 Reflection and propagation of laser pulse with a few cycles in medium with timedependent dielectric permittivity [8772-36]

V. A. Trofimov, I. V. Mishanov, M.V. Lomonosov Moscow State Univ. (Russian Federation)

877213 Numerical investigation of the Gaussian pulses propagating in optical fibers with refractive indices stochastically changed due to environmental conditions [8772-38]

L. Ladányi, R. Menkyna, J. Müllerová, Univ. of Žilina (Slovakia)

877214 Two-photon polymerization of diacrylate mesogen for producing polymer with patterned orientation structures [8772-39]

W. Zheng, W.-Z. Huang, National Sun Yat-Sen Univ. (Taiwan) 
877217 Determination of the uncertainty for phase noise delivered by an optoelectronic based system [8772-42]

P. Salzenstein, E. Pavlyuchenko, CNRS, Institut Franche Comté Electronique Thermique Optique Sciences et Technologie (France)

877218 Polarization properties of vector solitons generated by modulation instability in circularly birefringent fibers [8772-45]

E. A. Kuzin, Instituto Nacional de Astrofísica, Óptica y Electrónica (Mexico); A. Flores-Rosas, Univ. Guanajuato (Mexico); J. I. Peralta-Hernandez, B. A. Villagomez-Bernabe, B. Ibarra-Escamilla, A. Gonzalez-Garcia, Instituto Nacional de Astrofísica, Óptica y Electrónica (Mexico); O. Pottiez, Ctr. de Investigaciones en Óptica, A.C. (Mexico); M. Duran-Sanchez, Univ. Tecnológica de Puebla (Mexico)

8772 1B Frequency doubling of $\mathrm{cw}$ 1560nm laser with single-pass, double-pass and cascaded MgO:PPLN crystals and frequency locking to $R b \mathrm{D}_{2}$ line [8772-48]

S. Guo, J. Wang, Y. Han, J. He, Shanxi Univ. (China)

$87721 \mathrm{C}$ Highly nonlinear tellurite fiber with engineered chromatic dispersion for broadband optical parametric amplification [8772-49]

E. P. Samuel, T. H. Tuan, K. Asano, T. Suzuki, Y. Ohishi, Toyota Technological Institute (Japan)

8772 ID Second order optical nonlinear processes as tools to probe anomalies inside high confinement microcavities [8772-50]

M. Collette, N. Beaudoin, S. Gauvin, Univ. de Moncton (Canada)

$87721 \mathrm{E} \quad$ Experimental investigation of high power picosecond 1.06 $\mu \mathrm{m}$ pulse propagation in Bragg fibers [8772-52]

M. Jelínek, V. Kubeček, H. Jelĺnková, Czech Technical Univ. in Prague (Czech Republic); V. Matějec, I. Kašik, O. Podrazký, Institute of Photonics and Electronics (Czech Republic)

8772 1G Enhancement of optical nonlinearity of LCs with gold-nanoparticle-doped alignment layers [8772-54]

H.-C. Lin, National Formosa Univ. (Taiwan) and National Cheng Kung Univ. (Taiwan);

A. Y. G. Fuh, C. Y. Lin, M. S. Li, National Cheng Kung Univ. (Taiwan))

877211 Electronic structure of $\mathrm{KTiOAsO}_{4}$, a novel material for non-linear optical applications [8772-56]

$\vee$. V. Atuchin, AV Rzhanov Institute of Semiconductor Physics (Russian Federation);

O. Y. Khyzhun, V. L. Bekenev, A. K. Sinelnichenko, Frantsevych Institute for Problems of Materials Science (Ukraine); L. I. Isaenko, S. A. Zhurkov, Institute of Geology and Mineralogy, Siberian Branch (Russian Federation)

$87721 \mathrm{~L} \quad$ Optical propagation loss measurements in electro optical host-guest waveguides [8772-59] E. Nitiss, J. Busenbergs, M. Rutkis, Univ. of Latvia (Latvia)

$87721 \mathrm{M} \quad \mathrm{C}$-band amplification through fibre ring laser in generating multi wavelength [8772-60] M. N. Abdullah, S. Shaari, A. A. Ehsan, S. Menon, Univ. Kebangsaan Malaysia (Malaysia); M. N. Zainal Abidin, A. R. Zainal Abidin, National Metrology Lab. Malaysia (Malaysia) 
$87721 \mathrm{~N} \quad$ All-optical four-bit Toffoli gate with possible implementation in solids [8772-61]

G. Grigoryan, Institute for Physical Research (Armenia) and Russian-Armenian (Slavonic) Univ. (Armenia) V. Chaltykyan, E. Gazazyan, O. Tikhova, Institute for Physical Research (Armenia)

877210 Structural field of $\mathrm{K}_{2} \mathrm{Al}_{2} \mathrm{~B}_{2} \mathrm{O}_{7}$-family crystals [8772-62]

V. V. Atuchin, AV Rzhanov Institute of Semiconductor Physics (Russian Federation);

B. G. Bazarov, V. G. Grossman, Baikal Institute of Nature Management (Russian Federation); M. S. Molokeev, Institute of Physics, Siberian Branch (Russian Federation); Z. G. Bazarova, Baikal Institute of Nature Management (Russian Federation)

$87721 \mathrm{P} \quad$ Laser-induced incandescence of carbon surface: a method for temperature estimation [8772-63]

S. E. Zelensky, K. S. Zelenska, National Taras Shevchenko Univ. of Kyiv (Ukraine)

$87721 Q \quad$ Optimal doping of GaSe with isovalent elements [8772-64]

V. V. Atuchin, AV Rzhanov Institute of Semiconductor Physics (Russian Federation);

Y. M. Andreev, Institute of Monitoring of Climatic and Ecological Systems, Siberian Branch (Russian Federation); K. A. Kokh, Institute of Geology and Mineralogy, Siberian Branch (Russian Federation); G. V. Lanskii, A. V. Shaiduko, Institute of Monitoring of Climatic and Ecological Systems, Siberian Branch (Russian Federation); T. I. Izaak, V. A. Svetlichnyi, Tomsk State Univ. (Russian Federation)

Author Index 
Proc. of SPIE Vol. $8772877201-8$

Downloaded From: https://www.spiedigitallibrary.org/conference-proceedings-of-spie on 26 Apr 2023 Terms of Use: https://www.spiedigitallibrary.org/terms-of-use 


\title{
Conference Committee
}

\author{
Symposium Chairs
}

Jiri Homola, Institute of Photonics and Electronics of the ASCR, v.v.i

(Czech Republic)

Chris Edwards, Central Laser Facility, Science and Technology

Facilities Council (United Kingdom)

Mike Dunne, Lawrence Livermore National Laboratory (United States)

Ivo Rendina, CNR, Instituto per la Microelettronica e Microsistemi

(Italy)

Honorary Symposium Chair

Miroslav Miller, Institute of Photonics and Electronics of the ASCR, v.v.i

(Czech Republic)

Conference Chairs

Mario Bertolotti, Università degli Studi di Roma La Sapienza (Italy)

Joseph Haus, University of Dayton (United States)

Alexei M. Zheltikov, Lomonosov Moscow State University

(Russian Federation)

Conference Programme Committee

Kiyoshi Asakawa, Centro de Fisica de Materiales (Spain)

Javier Aizpurua, Centro de Fisica de Materiales (Spain)

Bruno Crosignani, CREOL, The College of Optics and Photonics,

University of Central Florida (United States)

Angela Maria Guzman, CREOL, The College of Optics and Photonics, University of Central Florida (United States)

Reinhard Kienberger, Max-Planck-Institut für Quantenoptik (Germany)

Yuri S. Kivshar, The Australian National University (Australia)

Geoffrey H. C. New, Imperial College London (United Kingdom)

Jan Perina Sr., Univerzita Palackého V Olomouci (Czech Republic)

Mark I. Stockman, Georgia State University (United States)

\section{Session Chairs}

1 Anisotropic Materials

Mario Bertolotti, Università degli Studi di Roma La Sapienza (Italy)

2 Nonlinear Materials

Ivo Rendina, Istituto per la Microelettronica e Microsistemi, CNR (Italy) 
3 Plasmons

Martti Kauranen, Tampere University of Technology (Finland)

4 Plasmonics

Javier Aizpurua, Centro de Fisica de Materiales (Spain)

5 Solitons

Javier Aizpurua, Centro de Fisica de Materiales (Spain)

6 Applications/Devices I

Mario Bertolotti, Università degli Studi di Roma La Sapienza (Italy)

7 Applications/Devices II

Alessandro Belandini, Università degli Studi di Roma La Sapienza (Italy)

8 Materials I

Mario Bertolotti, Università degli Studi di Roma La Sapienza (Italy)

9 Materials II

Joseph W. Haus, University of Dayton (United States) 